\title{
Bending Distortion Analysis of a Steel Shaft Manufacturing Chain from Cold Drawing to Grinding
}

\author{
VINICIUS WAECHTER DIAS, ALEXANDRE DA SILVA ROCHA, JULIANA ZOTTIS, \\ JUAN DONG, JÉRÉMY EPP, and HANS WERNER ZOCH
}

\begin{abstract}
Shafts are usually manufactured from bars that are cold drawn, cut machined, induction hardened, straightened, and finally ground. The main distortion is characterized by bending that appears after induction hardening and is corrected by straightening and/or grinding. In this work, the consequence of the variation of manufacturing parameters on the distortion was analyzed for a complete manufacturing route for production of induction hardened shafts made of Grade 1045 steel. A DoE plan was implemented varying the drawing angle, cutting method, induction hardening layer depth, and grinding penetration depth. The distortion was determined by calculating curvature vectors from dimensional analysis by $3 \mathrm{D}$ coordinate measurements. Optical microscopy, microhardness testing, residual stress analysis, and FEM process simulation were used to evaluate and understand effects of the main carriers of distortion potential. The drawing process was identified as the most significant influence on the final distortion of the shafts.
\end{abstract}

DOI: $10.1007 / \mathrm{s} 11661-016-3948-\mathrm{y}$

(C) The Minerals, Metals \& Materials Society and ASM International 2017

\section{INTRODUCTION}

THE part's geometry is well known as an important distortion potential. ${ }^{[1]}$ In particular for long parts, inhomogeneities of chemical composition, microstructure, or residual stresses can lead to large distortion. In the case of shafts, significant bending distortion can occur, which needs to be corrected by straightening and/ or grinding after the heat treatment. These expensive distortion correction processes are necessary to provide the final desired dimensional tolerances and account for an elevated part of the production costs. Therefore any effort to reduce distortion of automotive shafts is valuable when quality improvement and reduction of manufacturing costs can be achieved. Usually the effort to reduce distortion problems is done only by trying to improve single steps of the manufacturing chain (for example, by changing heat treating parameters). However, in the sense of the holistic view as proposed in "Distortion engineering", ${ }^{[1]}$ the manufacturing chain has to be seen as a whole and higher effort must be taken to extend the analysis to the maximum number of steps that can represent or contain possible causes of distortion.

VINICIUS WAECHTER DIAS, and JULIANA ZOTTIS are with the Metal Forming Laboratory, Federal University of Rio Grande do Sul, Av. Bento Gonçalves, 9500. Porto Alegre, CEP 91501-970, Brazil. Contact e-mail: vinicius.waechter@ufrgs.br ALEXANDRE DA SILVA ROCHA is with the PPGEM, Metal Forming Laboratory, Federal University of Rio Grande do Sul, Porto Alegre, Brazil JUAN DONG, JEREMY EPP, and HANS WERNER ZOCH are with the Division of Materials Science, Foundation Institute for Materials Science, Badgasteiner Str. 3, 28359 Bremen, Germany.

Manuscript submitted August 8, 2016.

Article published online January 13, 2017
Automotive shafts and other long components are often manufactured from cold drawn bars which are frequently obtained by a process called "combined cold drawing." It involves different manufacturing steps as uncoiling the wire rod, pre-straightening, shot blasting to remove the scale, drawing, cutting to a given length, polishing, and final straightening with crossed rolls. A previous project was carried out on the distortion potential of this specific manufacturing process. ${ }^{[2-4]}$ In that study, a statistical analysis of the whole manufacturing chain by Design of Experiment (DoE) has shown that the most significant influences on the distortion potential stored in these bars were the drawing process parameters (mainly the drawing angle) and the material batch as varied.

The present work aims at analyzing the transmission of these distortion potentials from the cold drawn bars (generated in the combined cold drawing) to the final product, as well as identifying new influences associated with the specific manufacturing steps. Thus, a "model shaft" with some geometric features of real shafts was established as final product. The experimentation then has been extended from cold drawing to further manufacturing steps, such as cutting of the cold drawn bars, machining to produce the shaft shape, induction hardening, and final grinding to correct the distortion. The focus of this study is to investigate how the main identified distortion potential carriers can be affected by process variations an influence distortion. Besides geometry, inhomogeneities of microstructure, hardness, and residual stresses are important carriers of distortion potential. Therefore, metallographic, microhardness, and residual stress analyses were carried out extensively. Additionally to the experimental work, numerical simulations using finite element method (FEM) were 
conducted with the focus on uncontrollable effects from the drawing process in the generation of inhomogeneous residual stresses. These non-influenceable effects are associated to process variations, like the shape of the bar entering the drawing tool or friction variations, which are very difficult to take into account in a normal DoE analysis.

\section{MATERIALS AND METHODS}

The investigated manufacturing process consists of two main parts: the combined drawing process and the shaft manufacturing steps (cutting, machining, induction hardening, and grinding). The investigated sample geometry was approached to realistic shafts by geometrical and length features, establishing what is called "model shaft" in this work, as seen in Figure 1(a). One important point for the whole analysis was the following of the orientation of the drawn bars by a visible mark and identification of the same positions in the final product (model shaft). This orientation system is shown in Figure 1(b), as well as the final geometry of the manufactured shafts (Figure 1(a)). The 0 deg reference angle is the upper position of the bars as they pass the drawing tool. The use of this reference system is extremely important for the comparison of results from different steps of the route, because it guaranties that all samples passed by the drawing tool in the same position. Thanks to this, inhomogeneous properties and distortion orientation can be related to the sample orientation in every single manufacturing step.

The material used was a Grade 1045 steel with the composition given in Table I. In the combined drawing process, a hot rolled steel wire rod (normalized state, presenting a banded structure of ferrite and pearlite) is pre-straightened by horizontal and vertical rolls, shot blasted, drawn, and cut to 6 meters long bars, and then polished and straightened by crossed rolls (PERC). This is all done in a continuous industrial process. These cold drawn and straight bars represent the starting material state for the model shaft manufacturing steps. The procedures for the shaft manufacturing followed some procedures as done by automotive manufacturing companies. The 6-m bars were cut into 410-mm (millimeters) pieces with the use of two different methods: shear cut, where the cut was performed with a guillotine assembly on a hydraulic press and the saw cut, which was performed with the use of a circular band saw machine. After the cut process, the samples were machined to 400 $\mathrm{mm}$ (to eliminate dimensional effects of the cut close to ends) and turned with the intention to replicate notches generally used on automotive shafts. The induction hardening process used a scan inductor with $22 \mathrm{~mm}$ diameter varying only the power to obtain different hardened layers. Other parameters, such as scan speed, frequency, and cooling rates that are known to affect distortion and residual stresses, ${ }^{[5]}$ were maintained equal to all pieces. All samples were tempered for 2 hours in $443 \mathrm{~K}\left(170{ }^{\circ} \mathrm{C}\right)$ in a conventional oven without atmosphere control. Finally, all the samples were ground with two different depths to decrease the bending generated after induction hardening. The whole manufacturing chain is presented in Figure 2 together with the parameter variations introduced in the process (inputs for a statistical analysis).

In order to evaluate the effects of each parameter on distortion, dimensional analysis after drawing, cutting, machining, induction hardening, and grinding were carried out with Zeiss Contura G2 3D and Leitz PMM 654 coordinate measuring machines. Prior to each measurement series, the dimensional probes from both machines were positioned in their original position at point $(0,0,0)$, and afterwards, a standard sphere was measured. Each shaft was clamped in the central plane with the $0^{\circ}$ line always pointing to the top. Twenty (20) contours each formed by 360 measuring positions were used to define the shape and size of the shafts. Then, the measured positions were fitted with a best fit circle by a least squares method. This procedure is given by Surm et $a l .{ }^{[6]}$ From these calculations, center positions of each circle were then known. Further data evaluation followed the procedure given by Frerichs ${ }^{[7]}$ using the projection of these centers on $x-y$ planes in the axial center of each bar, creating a vector with the length of this vector being the expression of the bars curvature. The distortion vector was used as output for the distortion analysis in the DoE.

As shown in Figure 2, the steps and parameters chosen for this DoE analysis were the drawing process with tool angles of 15 and $20 \mathrm{deg}$; cutting process by sawing and shear method; induction hardening layer depth of 1.2 and $2.2 \mathrm{~mm}$; and finally grinding depth of 100 and $200 \mu \mathrm{m}$ (micrometers). The DoE full factorial plan was carried out with dimensional measurements for the determination of bending of 5 samples for each parameter variation. A total of 80 samples were used in this statistical analysis of distortion. The software MINITAB ${ }^{\circledR}$ was used for the statistical evaluation of the DoE. The software uses Student's distributions (effects with unknown variance) for the evaluation of distortion data. Effects from manufacturing variables were calculated for a statistical significance of $\alpha=0.02$ (98 pct).

As residual stresses is an important carrier of distortion potential, surface residual stresses and depth profiles were measured at several positions by X-ray diffraction using $\mathrm{Cr}-\mathrm{K} \alpha$ radiation in the side inclination mode and evaluated by the $\sin ^{2} \psi$ method. More details were given in the previous works. ${ }^{[4,8]}$ Due to instrument limitations in covering measurements for the whole $400-\mathrm{mm}$ model shafts and to avoid cutting of the bars, measurements were performed at positions of 150,160 , 170,180 , and $190 \mathrm{~mm}$ of distance from the extremity of the shaft at the surface of the shafts without notches. The different cutting processes for shaft manufacturing were evaluated by residual stress measurements in the axial direction at distances of $0.5 \mathrm{~mm}$ up to $50 \mathrm{~mm}$ from the cutting surface for four reference angles $(0,90,180$, and 270 deg regarding Figure 2(b)). Additionally, residual stress (RS) and full width at half maximum (FWHM) depth profiles were obtained with the assistance of successive electrochemical layer and X-ray diffraction measurements. 

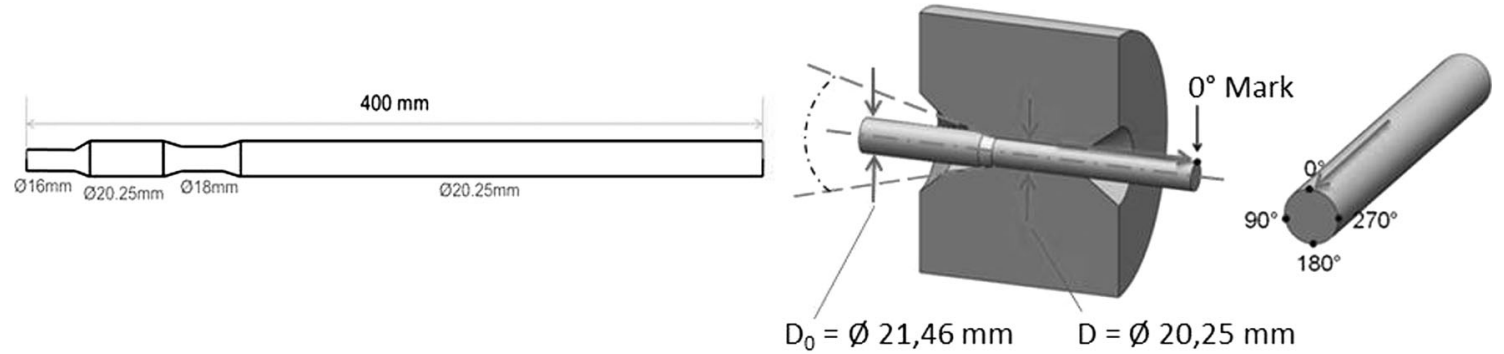

(a)

(b)

Fig. 1-(a) Shaft dimensions and (b) Guided system orientation.

Table I. Chemical Composition of the Steel Grade 1045 Used

\begin{tabular}{lccccccccc}
\hline Element & $\mathrm{C}$ & $\mathrm{Si}$ & $\mathrm{Mn}$ & $\mathrm{P}$ & $\mathrm{S}$ & $\mathrm{Cr}$ & $\mathrm{Mo}$ & $\mathrm{Ni}$ & $\mathrm{Cu}$ \\
\hline Mass pct & 0.48 & 0.23 & 0.87 & 0.02 & 0.03 & 0.09 & 0.02 & 0.05 & 0.16 \\
\hline
\end{tabular}



\begin{tabular}{|l|l|}
\hline \multicolumn{1}{|c|}{ Process/Parameter } & \multicolumn{1}{c|}{ Variations } \\
\hline Drawing/Drawing Angle & $15^{\circ}$ and $20^{\circ}$ \\
\hline Cutting/Cutting Method & Saw and Shear \\
\hline Induction Hardening/Hardened Layer & 1.2 and $2.2[\mathrm{~mm}]$ \\
\hline Grinding/Grinding Depth & 100 and $200[\mu \mathrm{m}]$ \\
\hline
\end{tabular}


Fig. 2-Investigated manufacturing route and varied process/parameters.

\section{RESULTS AND DISCUSSION}

As clarified in the introduction, the discussion in this paper will focus on the final manufacturing steps of shaft. The previous manufacturing steps of pre-straightening, shot blasting, and PERC affect shafts properties, ${ }^{[2,3]}$ and therefore were kept constant for a unique heat.

\section{A. Dimensional Results}

The curvature vectors of all investigated shafts were plotted in an $x-y$ diagram, allowing the observation of the orientation and the amplitude of the curvature after each step. Figure 3(a) and (b) present the curvature of the shafts before and after induction hardening and after grinding for two different drawing conditions (15 and 20 deg drawing angle). Before induction hardening, the curvature of the shafts are similar at low values for both drawing angles, as the bars are polished and straightened at the end of drawing before they are cut to the length and machined. The cutting and the machining processes did not influence significantly the curvature, and due to space limitations, this will be not discussed in this article. After the induction hardening process, the curvature values increase significantly for both drawing conditions, but with $15 \mathrm{deg}$ drawn angle, there is clearly a higher increase. Figure 3(a) shows that most of the distortion vectors (approximately 85 pct of these samples) after induction hardening turn to the same quadrant, while no preferred bending orientation can be observed for the 20 deg drawing angle (Figure 3(b)). The mean distortion increase after induction hardening was 189 and $81 \mu \mathrm{m}$, for 15 and 20 deg drawn bars, respectively.

The induction hardening layer depth influenced the curvature, with the surface hardened depth (SHD) of 1.2 $\mathrm{mm}$ presenting average distortions of $184 \mu \mathrm{m}$ and $66 \mu \mathrm{m}$ for 15 and 20 deg drawn bars, while the SHD $2.2 \mathrm{~mm}$ presented average distortions of 193 and $96 \mu \mathrm{m}$ for 15 and 20 deg drawn bars, respectively. The higher hardened depth generated higher curvatures for the different drawing angles. A DoE specific for the distortion after induction hardening was presented in a previous work where more details are given by Dong et al. ${ }^{[9]}$ After the grinding process, the curvature vectors (circle marks) show a large reduction in their magnitude, but the remaining curvature is still slightly higher for the $15 \mathrm{deg}$ drawing angle than for $20 \mathrm{deg}$. It is also possible to 


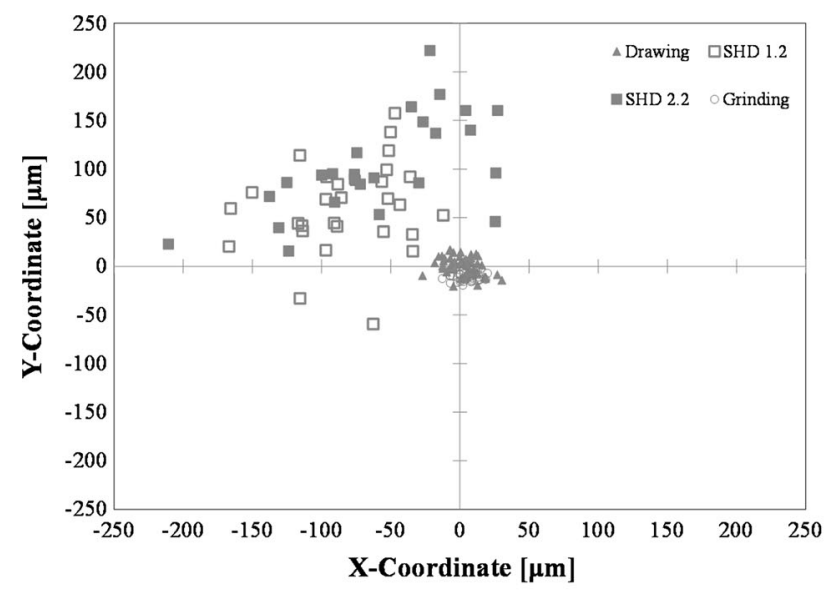

(a)

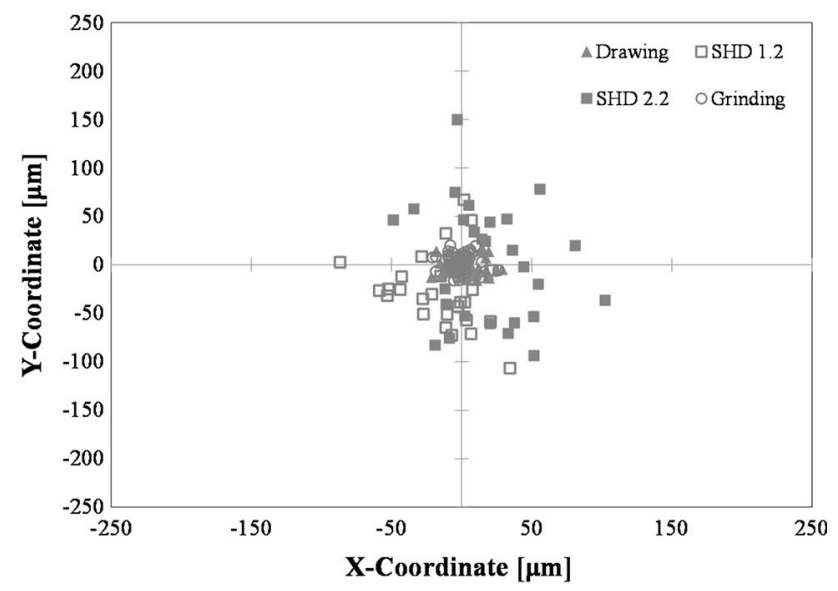

(b)

Fig. 3-Curvature vector changes in before and after Induction hardening and grinding for (a) 15 deg drawing tool and (b) $20 \mathrm{deg}$ drawing tool.

observe comparing curvature vectors in Figure 3(a), that the distortion vectors show a direction change at about 180 deg after grinding compared with the direction after induction hardening. This can be explained by the higher removal of material in surface areas of the bar which are convex in relation to the grinding wheel. For the $100-\mu \mathrm{m}$ removed layer, the resulting curvature was 14 and $10 \mu \mathrm{m}$, respectively, for 15 and $20 \mathrm{deg}$ drawn bars, while for the $200-\mu \mathrm{m}$ removed layer, the respective values were 14 and $16 \mu \mathrm{m}$. The influence of removing 100 or $200 \mu \mathrm{m}$ was not significant for the amount of remaining distortion observed, showing that $100 \mu \mathrm{m}$ would be already enough to obtain a mean curvature value below $15 \mu \mathrm{m}$.

It is also clear that the results for all bars drawn in the 15 deg drawing tool presented a preferred distortion direction. Previous investigations have shown that a 15 deg drawing tool angle potentially generates lower residual stress differences between core and surface ${ }^{[4,10]}$ and from that a lower distortion after heat treatment would be expected. However, this was not the observed effect, showing that uncontrollable variables of the combined cold drawing process are stronger than this drawing angle tool variation.

\section{B. Cold Drawn Bars}

Centerline segregation has been found in the rods. The chemical composition in the different points presents a difference in the carbon content of up to 0.2 Mass-pct higher in the core segregation. In the macroetched (Nital 4 pct) cross sections presented in Figure 4, it is possible to observe that the center segregations present differences in their morphology and positioning for both samples even though they were originated from the same heat and the same wire rod. These variations might represent a certain distortion potential which could be released during heat treatment, ${ }^{[11]}$ as the varying properties of the different zones can lead to changes in stress generation, phase transformation kinetics, etc. ${ }^{[12]}$

The hardness results for the samples with drawing angle of 15 and $20 \mathrm{deg}$ present differences close to the surfaces between both conditions. The hardness measurements indicate a higher hardness near to the surface for the samples drawn with the angle of $20 \mathrm{deg}$, around $290(+/-15) \mathrm{HV} 1$. The $15 \mathrm{deg}$ drawing angle resulted in hardness values around $280(+/-10) \mathrm{HV} 1$, while the average core hardness was $260(+/-8) \mathrm{HV} 1$. This was identified to be a consequence of a stronger plastic deformation of the near-surface region in the process with 20 deg drawing angle compared to the drawing angle of $15 \mathrm{deg}$. Additionally, it could be observed that variations of the near-surface hardness are present over the circumference and over the length, indicating that local inhomogeneities are present in the drawing process and in the material.

The residual stresses in the surface of the samples before the heat treatment presented values close to -250 $\mathrm{MPa}$ for the $15 \mathrm{deg}$ sample and $-100 \mathrm{MPa}$ for the 20 deg sample and are distributed non-homogeneously along the surface reference points (scattering in the range of $50 \mathrm{MPa}$ ). These results are very similar to previous results presented by Hirsch et al. ${ }^{[4]}$ who studied the same combined drawing process for other Grade 1045 steel batches produced.

\section{Cutting Method}

The cutting method was investigated in order to evaluate the introduced distortion potential. The residual stress results concerning the different cutting methods show that after a distance of $30 \mathrm{~mm}$, effects of both types of cutting fade away. Figure 5(a) displays that the shear cutting leads to a complex distribution of residual stresses up to $30 \mathrm{~mm}$ for the shear cut bars. Large differences can be observed at different positions around the circumference, which is directly related to the movement of the shearing tool (entering at $0 \mathrm{deg}$ ). Results presented in Figure 5(b) for the saw cut samples show only a small effect compared to the shear cut, with a maximum affected zone $3 \mathrm{~mm}$ from the cutting edge. Additionally, microhardness average results presented strongly elevated values for the shear cut samples close to the cut surface (around $350 \mathrm{HV} 1$ for both drawing angles) due to pronounced plastic deformation. For the saw cut samples, the average hardness values found were 




(a)

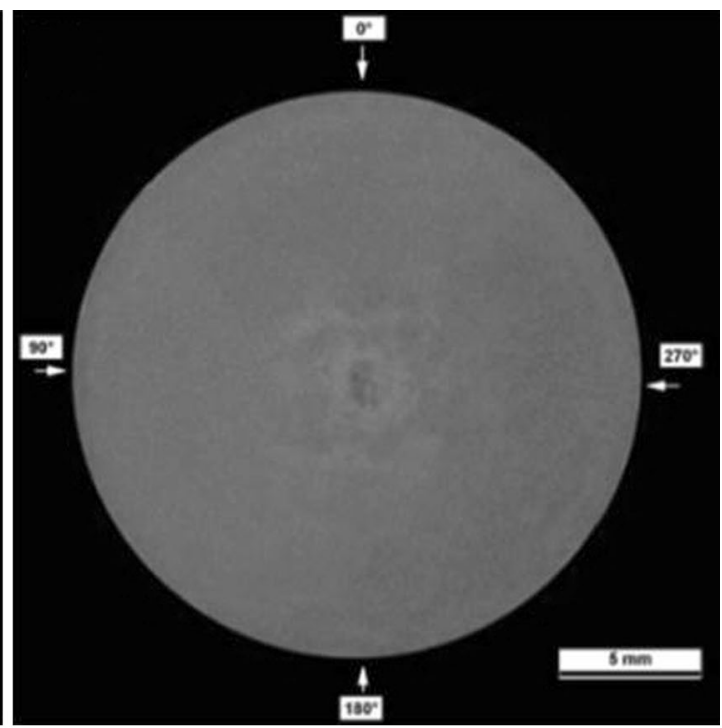

(b)

Fig. 4-Macro-etched (Nital 4 pct) cross-section pictures from samples taken from $(a)$ beginning and $(b)$ end of the same wire rod.

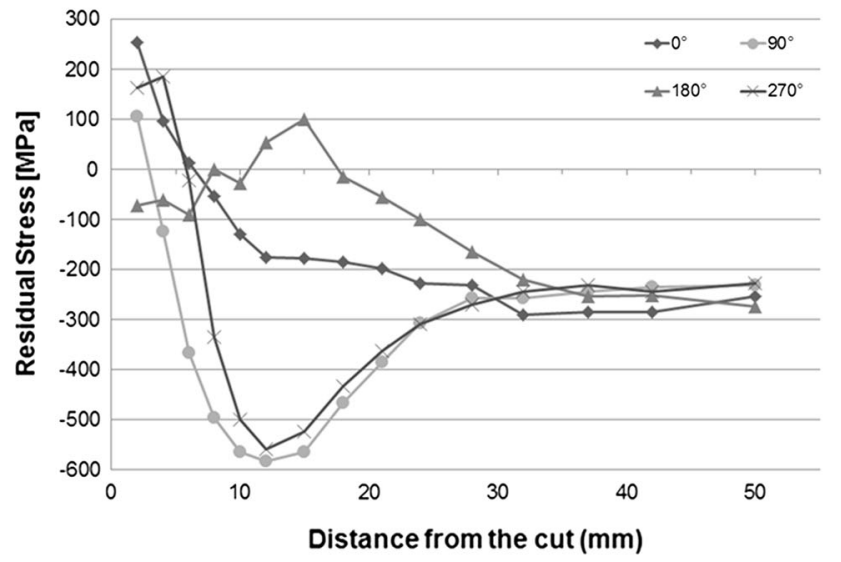

(a)

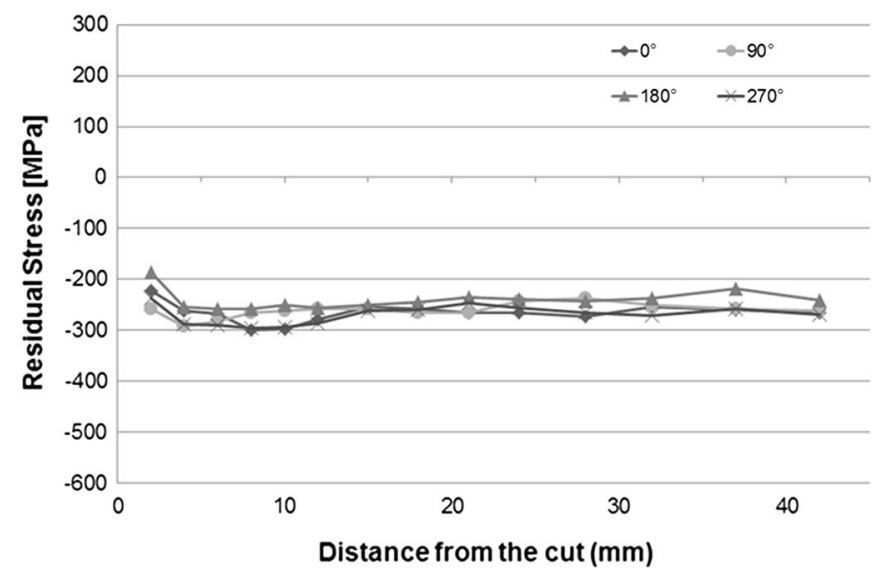

(b)

Fig. 5-Axial residual stresses after different cutting methods: (a) Shear and (b) Saw.

very close to the core hardness of the bars (around 280 HV1).

The cutting step was not identified to be a significant distortion potential. Even if a large zone is strongly influenced by shear cutting (around $30 \mathrm{~mm}$ ), machining operation removed a large portion of the influenced zone $(10 \mathrm{~mm})$, leading to only small distortion potential remaining (5 pet of the bar length).

\section{Induction Hardening}

The residual stress results were analyzed for both drawing angles and the different surface hardened depths (SHD). Figure 6 presents the residual stress and full width at half maximum (FWHM) profiles for samples with $15 \mathrm{deg}$ drawing angle and the SHD of 2.2 (Figure 6(a)) and $1.2 \mathrm{~mm}$ (Figure 6(b)). The residual stress profile for the sample with SHD of $2.2 \mathrm{~mm}$ presented varying values in the measured depths of 0 (surface) and $50 \mu \mathrm{m}$, which was not observed in the subsequent measured depths (up to $300 \mu \mathrm{m}$ ). In the sample with SHD of $1.2 \mathrm{~mm}$, the observed scattering is concentrated only in the surface position $(0 \mu \mathrm{m})$. The same scattering pattern (same positions) can also be observed on the FWHM with a smaller amplitude. This variation can be related to decarburizing and retained austenite formation, which is common in this type of process. The larger variations in the $2.2-\mathrm{mm}$ hardened shaft can also be related to the coarser martensite needles due to the higher austenitizing temperature necessary for the hardening depth ${ }^{[5]}$ : $1123 \mathrm{~K}\left(850^{\circ} \mathrm{C}\right)$ for $1.2 \mathrm{~mm}$ and $1243 \mathrm{~K}\left(970{ }^{\circ} \mathrm{C}\right)$ for $2.2 \mathrm{~mm}$.

Although the induction hardening process should be an axis-symmetric and homogeneous procedure, inhomogeneities present in the properties before treatment and previously introduced distortion potential can be 



(a)


(b)

Fig. 6-Axial residual stresses and FWHM after induction hardening for samples drawn with 15 deg die tool and SHD of (a) 2.2 mm and (b) $1.2 \mathrm{~mm}$ for the removed layers (50 to $300 \mu \mathrm{m}$ ).

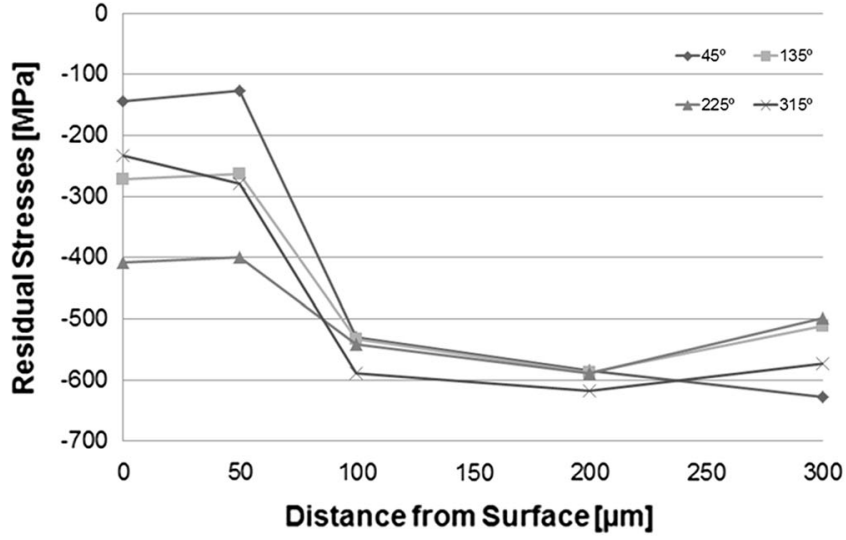

(a)

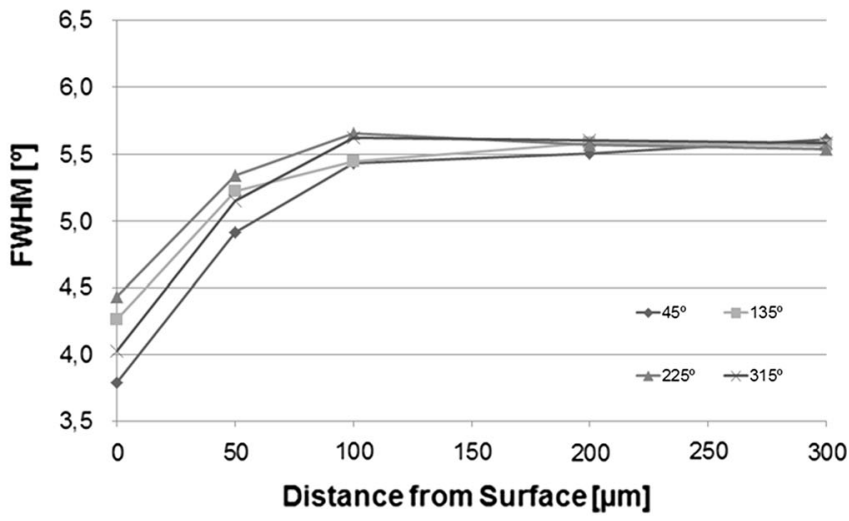

(b)

Fig. 7- (a) Axial residual stresses and (b) FWHM after grinding for the sample with SHD of $2.2 \mathrm{~mm}$ and distances from surface of 50 to 300 $\mu \mathrm{m}$.

present in the samples. The inhomogeneities are not only in terms of microstructure, but also mainly of residual stresses and cold working due to the previous processes, as shown along this paper. During the induction hardening, the distortion potentials can be released via relaxation of residual stresses together with recovery and 
recrystallization, transformation induced stresses, transient thermal stresses, and volume changes leading to shape and size changes (distortions). If non-symmetric (non-homogeneous) distortion potential was introduced into the sample from the manufacturing process, a bending of the bars can occur during induction hardening even for a perfectly symmetrical process. This would cause heating differences accordingly with the distance from the inductor to the shaft, and therefore additional inhomogeneities of the hardened layer. Higher inhomogeneities (released distortion potential), can result in distortion. ${ }^{[5]}$ This higher amount of released inhomogeneities might also explain why thicker hardened layers lead to higher distortions.

\section{E. Grinding After Induction Hardening}

The residual stress results for the grinding process are presented in Figure 7(a) for a shaft with 15 deg drawing angle SHD of $2.2 \mathrm{~mm}$ and grinding depth of $200 \mu \mathrm{m}$. Inhomogeneity of the residual stresses over the circumference (over $250 \mathrm{MPa}$ differences) and a considerable reduction on the compressive stress due the thermo-mechanical influence are observed compared with the results of induction hardening (see Figure 6) on the measuring depths of 0 and $50 \mu \mathrm{m}$. This effect can be confirmed by the analysis of the FWHM results, presented in Figure 7(b), where the affected area (measured depths of 0 and $50 \mu \mathrm{m}$ ) presented a clear reduction in the values for the same depths (0 and $50 \mu \mathrm{m})$.

\section{F. DoE Analysis}

The DoE results presented as Pareto chart in Figure 8(a) were obtained using the final distortion of the shafts. The analysis of the curvature results shows that the drawing process variation is the most significant factor with a confidence of 98 pct. A previous DoE analysis ${ }^{[9]}$ before the grinding process also presented the drawing variations as the most significant factor. The interaction between drawing and cutting also appears significant, as well as interaction of cutting and induction hardening. The grinding process represents the last of the significant parameter. The higher influence of the drawing process as seen from the measured data was already expected and is confirmed by the DoE. In a previous research, ${ }^{[4]}$ drawing was also found as the most significant distortion potential in cold drawn bars. This result is now confirmed when the process is extended to the manufacturing of a shaft.

Figure 8(b) presents how each parameter individually influences the distortion. These results are in agreement with all the results presented so far. The saw and shear cut presented very similar effects on mean distortion values. Although the saw cut presented lower influence on the distortion potentials, this cut presented higher influence on distortion. This can be explained by the fact that once the distortion potentials on the shear cut are higher but localized on the end of the shafts, they may lead to different distortion behavior at the end of the bar (last 10 to $20 \mathrm{~mm}$ ) reducing the global distortion values and acting as a compensation potential instead.

\section{G. Drawing Uncontrollable Variables Analysis Through FEM}

The results presented by the DoE analysis in the Figure 8(a) have shown that the drawing process has the most significant influence on distortion after grinding. Other possible variables generating inhomogeneities of residual stresses and cold working in the drawn material should be taken into account, once the drawing angle of $15 \mathrm{deg}$ led to higher distortion values which were not expected due to the lower stress gradients in the bars. ${ }^{[4]}$ These variables were called uncontrollable variables. The pre-straightening process affects the surface residual stress distribution obtained after drawing even when shot blasting was applied before. The bending effects left by pre-straightening will be superimposed to the drawing residual stresses. ${ }^{[13]}$ Misalignments between bar and tools, changes in friction coefficients, microstructure heterogeneities were also identified as uncontrollable variables. In order to determine their influence, two of those uncontrollable variables were investigated in terms of residual stresses resulting after drawing by means of FEM numerical simulations. The effects of segregation in the bars, and the effect of misalignments between bar and drawing tool and the fact that bars can enter the drawing tool with some curvature.

The numerical simulations using finite element method (FEM) through Deform ${ }^{\mathrm{TM}}$ and Simufact.forming $\mathrm{GP}^{\circledR}$ software were carried out to understand effects of possible uncontrollable parameter variations during drawing on the residual stress distributions in the bars used for shaft manufacturing. For the FEM numerical simulation of the drawing process, a hexahedral mesh was defined to be the most appropriate after validation tests using different shape of the elements (mesh type). In order to decrease computational time, simulation was restricted to a physical length of $200 \mathrm{~mm}$. Material true stress $v s$ true strain curve data was determined from own experimental investigations. ${ }^{[10]}$

Metallurgical analyses of the investigated material showed that a center segregation line is present close to the core of the bars but with varying position and extension along the length of the material (e.g., Figure 4 shows different positions from the same wire rod). These segregations show different properties than the rest of the material due to higher carbon and manganese content. ${ }^{[9]}$ In order to simulate this effect, a different material at the core of the sample bar was defined in the simulation. In a first approximation, the steel Grade 1060 was chosen from the available possibilities in the database of the software Simufact.formingGP ${ }^{\circledR}$ to represent this richer carbon zone. As segregation lines are non-homogeneously distributed over the cross section of steels, three different positions for these lines were tested, which are nominated as follows:

- Center: At the center of the bar;

- Shifted 1: 1-mm eccentricity;

- Shifted 2: 2-mm eccentricity. 




(a)

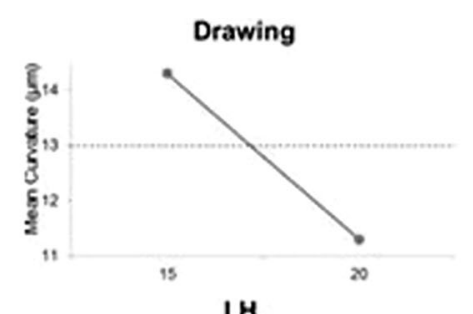

I.H.

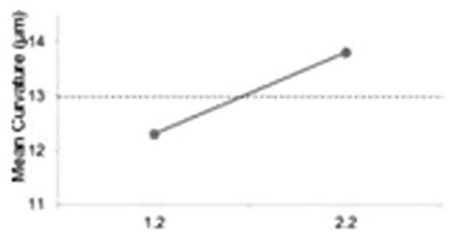

(b)

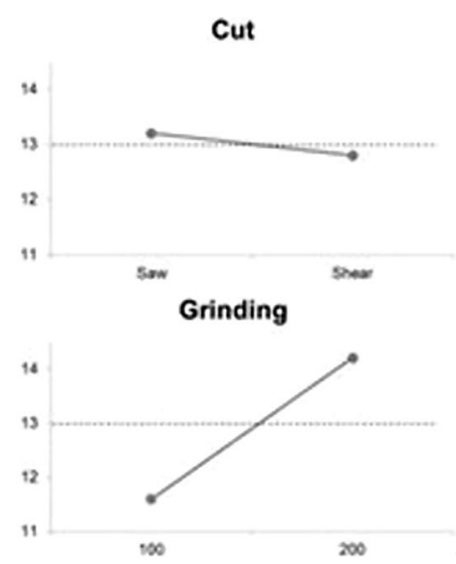

Fig. 8- (a) Pareto chart of the standardized effects for third-order interactions with confidence factor of 98 pct $(\alpha=0.02)$; $(b)$ Main effects plot for curvature.

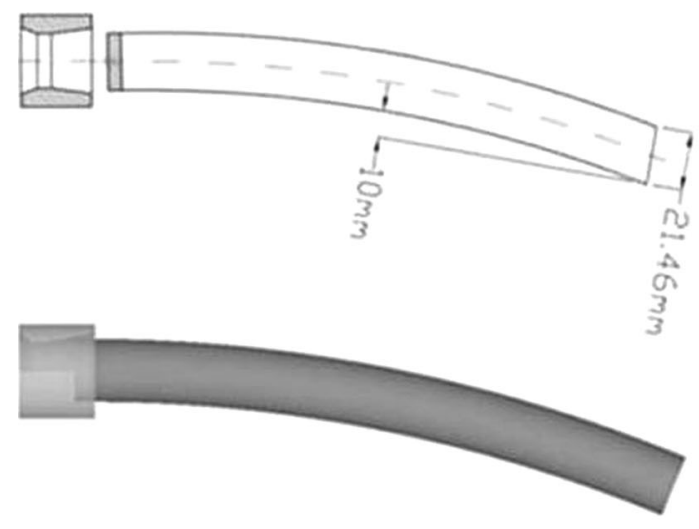

(a)

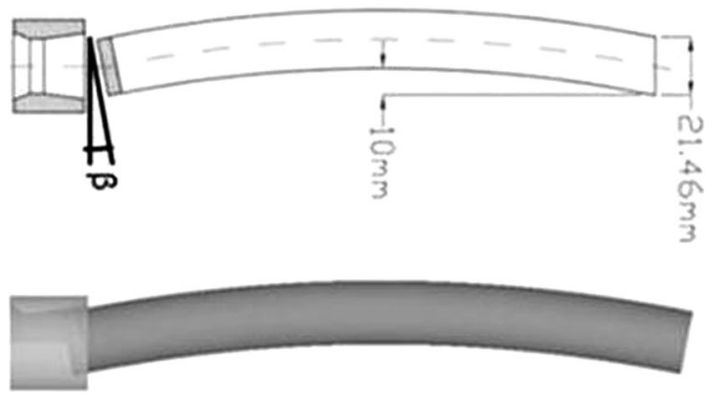

(b)

Fig. 9-Schematic drawing of (a) curved bar with the entrance aligned with the tool and $(b)$ curved bar with the misalignment angle $\beta$.

A misalignment and a curvature of the bars entering the drawing tool were further identified as potentially present in the drawing process. Indeed, the uncoiled rod is pre-straightened before entering the drawing tool. However, even after this process, the material still present a remaining curvature while entering the drawing tool. Misalignments of the bar regarding the drawing tool are also possible. These considerations were proofed by simulations aiming at analyzing these influences on the residual stress distributions. Therefore the model for simulation included different combinations as follows:

- Aligned: Curved bar aligned with the drawing tool (Figure 9(a));

- Misaligned: Curved bar combined with a misalignment between the bar and the drawing tool (Figure 9(b)).

The die angle considered for simulation was $20 \mathrm{deg}$, while the misalignment angle $(\beta)$ was chosen as $1 \mathrm{deg}$. Bars with a curvature of $10 \mathrm{~mm}$ with a length of $200 \mathrm{~mm}$ were considered. The curvature value was obtained through the measurement of the wire rod bar, as an extreme case (as if the pre-straightening had no effect). The software used to perform those simulations was Deform $^{\mathrm{TM}}$. The influence of the sample curvature entering the drawing tool and of the sample misalignment with the drawing tool was analyzed in several steps (Figure 10(a)). First, an idealized case was considered, using a perfectly straight bar, perfectly aligned with the tool (Standard). A perfectly symmetric residual stress distribution was obtained over the cross section after drawing with high tensility at the surface and high compression in the center. Neutron diffraction measurements of residual stress distribution in the cross section of a drawn bar previously published by Hirsch ${ }^{[4]}$ show that non-centered, asymmetric residual stress state is present (Neutrons).

Simulation results using a curved bar entering the tool without misalignment (Aligned) show an asymmetric residual stress distribution which comes closer to the measured profile. This is an indication that the sample curvature can play a significant role in the residual stress generation during drawing and so introduces a certain distortion potential in the material. The same simulation but using a misalignment of the entering bar does not 


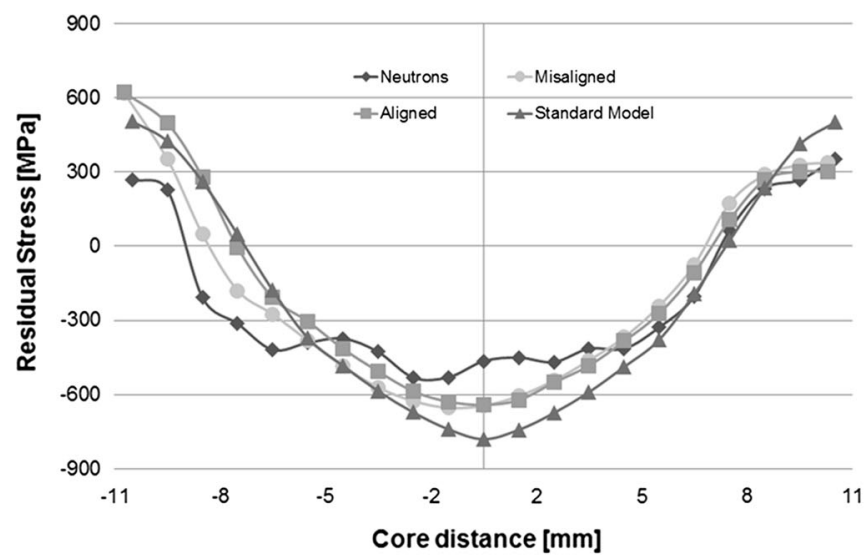

(a)

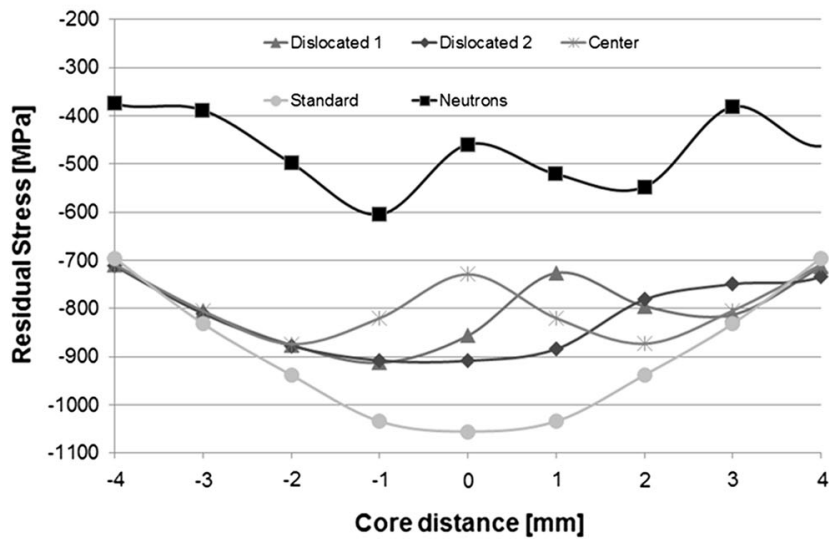

(b)

Fig. 10-Residual stress profiles for the simulated conditions compared with neutron measurements (Neutrons) and the reference simulation (Standard) for $(a)$ curved bar with misaligned (Misaligned) and aligned (Aligned) tool, and $(b)$ Variation of positions of the segregation line.

lead to large changes in the residual stress distribution, so that this parameter does not appear as of primary importance concerning the introduction of distortion potential.

The second simulation analysis was focused on the effect of the center segregation on the residual stress state after drawing. Figure 10(b) presents a comparison between the measured residual stress profile and simulation results without and with the use of a segregation line close to the core $(-4$ to $+4 \mathrm{~mm})$, by varying the positions of the line (Center; Shifted 1 and Shifted 2). The measured profile presents a non-monotone and non-symmetric distribution close to the center which cannot be observed in the idealized simulation without taking into account the core segregation. The introduction of the center segregation in the simulation leads to a strong modification of the simulation result showing a local compressive residual stress minimum in the center of the segregation line. A variation of the position of the segregation line leads to a related shift of this local minimum (Shifted 1 and Shifted 2).

Although the absolute residual stress values of experimental and simulation results still present a gap, the introduction of center segregation and of a slight shift from the center seems to reproduce similar variations in the distribution as observed by neutron measurements. This is an indication that the center segregation can be of importance in the generated residual stress distribution after drawing which then can represent a certain distortion potential. As the position and the extension of the segregation line can vary along the length or the rod, varying distortion potential can result and lead to scattering in the observed final distortion (Figure 4).As indicated by the simulation results, the variables influencing the drawing process cannot be only attributed to the drawing tool geometry (drawing angle, for example). Parameters such as friction coefficient, previous curvature of the bar, and microstructure can also influence the drawing process. In order to investigate and separate the contribution of each single parameter in the drawing process, detailed investigations under defined conditions should be performed in the future.

\section{CONCLUSIONS}

The combined cold drawing has the highest influence on the observed distortion with a confidence factor of 98 pct.

Although in the drawing process the intentionally varied parameter was the drawing angle, the effects observed cannot be attributed only to the tool geometry, since "uncontrollable variables" related to the combined cold drawing process were identified.

In the simulation, when regions with higher carbon content (segregation lines) and a bended bar entering the drawing tool are included in the model, a better agreement between simulated and measured RS was found.

Although the material state (microstructure and chemical composition) was not included in the intentionally changed process variables, they might be an important distortion potential, as the segregation zone shape and position vary along the wire rod, leading to the scattering of the distortion values.

The grinding process was able to decrease the distortion with both layer removal depths. However the resulting distortion vector was then oriented to a direction about $180 \mathrm{deg}$ in relation to the orientation after induction hardening.

\section{ACKNOWLEDGMENTS}

The authors would like to thank the funding agencies CAPES and CNPq from Brazil and DFG from Germany for the financial support for this work within the framework of the BRAGECRIM program and the HZB Berlin for the neutron measurements. 


\section{REFERENCES}

1. H W. Zoch: Proceedings 2nd Int. Conf. on distortion engineering IDE, 2008, Bremen, Germany, pp. 3-12.

2. Rocha AS, Nunes RM, de Souza, TF, Soares CAT, Stephan H, Tekkaya AE, Hirsch TK.: 3rd Int. Conference on distortion engineering IDE, 2011, Bremen, Germany; pp. 229-38.

3. A.S. Rocha, R.M. Nunes, and T.K. Hirsch: Mater. Res., 2012, vol. 15 , pp. 266-76.

4. T.K. Hirsch, A. da Sliva Rocha, and R.M. Nunes: Metall. Mater. Trans. A, 2013, vol. 44A, pp. 5806-16.

5. Rudnev V, Totten GE.: Handbook Volume 4C: Induction heating and heat treating, 2014.

6. H. Surm, O. Kessler, F. Hoffmann, and P. Mayer: Int. J. Mater. Prod. Technol., 2005, vol. 24 (1-4), pp. 270-81.

7. F. Frerichs, D. Landek, T. Lubben, F. Hoffmann, and H.W. Zoch: Mater. und Werkst., 2006, vol. 37 (1), pp. 63-68.
8. J. Dong, J. Epp, A.S. Rocha, R.M. Nunes, H.-W. Zoch, and R. Wimpory: HTM J. Heat Treat. Mat, 2014, vol. 69, pp. 348-59.

9. C.A.T. Soares, J. Zottis, T.F. Souza, L.M. Freitas, T. Colombo, and A.S. Rocha: Estud. Tecnológicos Eng., 2013, vol. 9 (2), pp. 54-62.

10. Prinz C, Hunkel M, Clausen B, Hoffmann, F, Zoch H-W: Proceedings 2nd Int. Conf. on distortion engineering IDE, 2008, Bremen, Germany, pp. 61-68.

11. Hunkel M.: Proceedings 2nd Int. Conf. on distortion engineering IDE, 2008, Bremen, Germany, pp. 399-410.

12. J. Dong, J. Epp, A. da Sliva Rocha, R.M. Nunes, and H.-W. Zoch: Metall. Mater. Trans. A, Phys. Metall. Mater. Sci., 2016, vol. 47A, pp. $877-88$

13. Rocha AS, Nunes RM, Metz M, Hirsch TK: Proceedings 2nd Int. Conf. On Distortion Engineering IDE, 2008, Bremen, Germany, pp. 3-12. 